\title{
Reflexiones en el cuarto año del programa de formación de médicos especialistas para la atención primaria de salud
}

\author{
CARLOS MONTOYA-AGUILAR ${ }^{(1)}$
}

\section{EL SERVICIO NACIONAL DE SALUD Y LA ATENCIÓN PRIMARIA (APS)}

La idea del Programa surgió en 1956 frente a las perspectivas derivadas de la creación del Servicio Nacional de Salud de Chile y de la posibilidad de que la Universidad de Chile ofreciera a los estudiantes de las carreras asociadas a la salud una enseñanza acorde con la doctrina del Servicio.

Ya en 1945 el Servicio Nacional de Salubridad, uno de los organismos que después se fusionaron en el SNS, había creado centros ambulatorios de salud llamados Unidades Sanitarias. El Dr. Gustavo Molina resumía de este modo las orientaciones derivadas de la experiencia de hacer atención primaria desde la Unidad Sanitaria de Antofagasta: " el objetivo primero de la actividad de salubridad -decíaes la prevención de la enfermedad y en todas ellas la base es el saneamiento ambiental. Sobre esta base se construye la superestructura de la higiene materno infantil, cultura sanitaria, nutrición, prevención de enfermedades no infecciosas... La acción sanitaria obliga a una permanente iniciativa local que no espera directivas de Santiago para actuar, sino que ajusta con agilidad sus programas para servir oportuna y sistemáticamente las necesidades de la comunidad que atiende. Esto da absoluta primacía a la calidad del trabajo sobre la cantidad... El conocimiento y contacto continuado con un sector de población es indispensable.
La concurrencia de la persona a cualquier servicio médico-preventivo-asistencial debería ser una oportunidad preciosa para abordar en ella todos los problemas de salud: por muchos años la condición de estar enfermo ofrecerá un momento y motivo valioso para hacer efectiva prevención... La familia como unidad debe ser el sujeto de toda actividad tendiente a la protección y fomento de la salud. Olvidando la concepción unitaria de la salud pública, se tiende a dividir artificialmente a la población en "problemas", tales como tuberculosis, o enfermedad venérea o madre y niño... Ningún programa alcanzará debida eficacia si no se abordan en conjunto esos problemas en el seno de cada hogar. El sujeto de la labor es uno: la población total agrupada en las unidades familiares y en los barrios en que la vida se desenvuelve."

Años más tarde, la Dirección del Servicio Nacional de Salud decía: "Sanidad y asistencia son inseparables"... "La Medicina es una y se

Tabla 1. Número de especialistas en formación, según año de ingreso al Programa.

\begin{tabular}{|c|c|}
\hline Año & Número \\
\hline 2008 & 25 \\
\hline 2009 & 67 \\
\hline 2010 & 96 \\
\hline 2011 & 111 \\
\hline Total & 299 \\
\hline
\end{tabular}

(1) Profesor titular de Salud Pública, Universidad de Chile. Alberto Henckel 2337. Providencia. Santiago. Chile. carlerne@gmail.com 
realiza mediante la integración de sus funciones de reparación, prevención y fomento de la salud. Su objeto no es ya el paciente fragmentado por la especialización; es el hombre en su integridad biofísica y social; es la persona.... es el ser social.... en recíproca acción y reacción con su medio. El SNS constituye la expresión objetiva de esas tendencias. La integración es su sello distintivo...." "La complejidad de los factores que condicionan el estado de salud obliga al trabajo en colaboración de diversos grupos de profesionales con distinta formación....." "La estructura local del SNS está destinada a realizar a través de los Centros de Salud una política integral hacia el fomento, la protección y la reparación de la salud." ${ }^{2-4}$

Tabla 2. Número de especialistas en formación en el año 2011, según las universidades en que cursan dicha formación.

\begin{tabular}{|l|c|}
\hline \multicolumn{1}{|c|}{ Universidad } & Número \\
\hline U. de Chile & 175 \\
\hline U. de Santiago de Chile & 39 \\
\hline U. del Desarrollo * & 32 \\
\hline U. D. Portales * & 7 \\
\hline U. de Valparaíso & 20 \\
\hline U. de Concepción & 16 \\
\hline U. de La Frontera (Temuco) & 6 \\
\hline U. Austral (Valdivia) & 4 \\
\hline Total & 299 \\
\hline
\end{tabular}

* privada.

En 1958-59 se organizó, en el seno de la Cátedra de Higiene y Medicina Preventiva y Social del Profesor Benjamín Viel y en convenio con el Servicio Nacional de Salud, el Centro de Demostración de Medicina Integral $(\mathrm{CDMI})^{5}$. Se trataba de un consultorio ubicado en la comuna de Quinta Normal, en el cual un equipo formado por especialistas básicos que compartían su tiempo entre hospital y atención ambulatoria, junto con enfermeras, asistentes sociales, matronas y auxiliares atendía a toda la población residente en un sector de doce mil habitantes. Ahí se hacía docencia teórica y práctica de salud pública a estudiantes e internos de distintas carreras relacionadas con la salud. Se hacía investigación y se siguieron desarrollando los conceptos expresados por los precursores, con aplicación progresiva en la planificación, en el trabajo familiar y comunitario, en la intersectorialidad, en el control de la mortalidad y morbilidad del niño y de sus causas, en la lucha contra el alcoholismo y sus consecuencias. Objetivos similares perseguían otros grupos docentes en diversas áreas de Santiago, en Concepción y en Valparaíso. El Profesor Gustavo Molina, citado más arriba, se esforzaba por llevar la epidemiología y los principios de prevención y fomento a la sala de hospital, en el Servicio del Profesor de Medicina Dr. Rojas Villegas ${ }^{6}$. El Profesor Amador Neghme y su grupo convergían desde su base en la Parasitología; lo propio ocurría desde la Microbiología y desde la Psiquiatría.

Gran parte de la labor de los consultorios urbanos del SNS, y también la de los hospitales rurales -la Medicatura General de Zona se inició en 1955 - estuvo impregnada de las ideas trabajadas en la Dirección del SNS, en la escuelas universitarias de la salud, en la Escuela de Salubridad y en la Oficina Sanitaria Panamericana. Así se afirmaron las bases para reducir la mortalidad infantil y materna, las enfermedades trasmisibles, la desnutrición y la incultura sanitaria.

Se estaba haciendo -en Chile- "Atención Primaria" pertinente y efectiva, con los nombres de atención integral, de atención comunitaria o de salud pública. La Reunión de Alma Ata y la Reforma de Salud del Brasil ocurrieron veinte años después, en 1978, y dieron expresión social y política a principios similares, acuñando el nombre de Atención Primaria de Salud.

\section{REDUCCIÓN DE RECURSOS. MUNICIPALIZACIÓN DE LA APS. EL DEBATE}

A partir del brusco cambio de régimen de septiembre de 1973, se redujeron los recur- 
Tabla 3. Número de especialistas en formación en el año 2011, según especialidad.

\begin{tabular}{|l|c|}
\hline \multicolumn{1}{|c|}{ Especialidad } & Número \\
\hline Medicina Interna & 121 \\
\hline Pediatría & 79 \\
\hline Obstetricia y Ginecología & 50 \\
\hline Psiquiatría & 46 \\
\hline Medicina familiar & 1 \\
\hline Neurología & 2 \\
\hline Total & 299 \\
\hline
\end{tabular}

sos, las acciones y la organización del SNS, así como el desarrollo de la salud pública. Algunos directivos y profesores, apoyados en la Declaración de Alma Ata y en la colaboración de la Unicef mantuvieron -no sin dificultades el control de la mortalidad infantil, con acciones como la creación de nuevos consultorios, la continuidad del programa de alimentación complementaria y -cosa muy importante- la auditoría de cada muerte infantil y materna.

En 1980 comenzó la municipalización de la atención primaria. Ésta se completó durante el régimen siguiente, con el Estatuto de 1995, en medio de una ardua discusión. En la Sociedad Chilena de Salubridad se sostenía que los municipios tienen funciones propias, tradicionales y muy importantes en el mejoramiento de la salud. Éstas son: el cuidado del medio ambiente y de la seguridad local; la información a la población acerca del tema de salud; la promoción de salud a través del apoyo a las organizaciones sociales, y la facilitación del contacto entre los establecimientos de salud y la población. Pero no corresponde que se encargue a las municipalidades la administración de establecimientos de atención de salud, por las siguientes razones: no tienen la capacidad técnica pertinente; las autoridades y funcionarios cambian en cada elección, con la correspondiente discontinuidad y desorganización de las políticas; hay una influencia muy directa de la política partidista y de los grupos de interés locales; hay una enorme desigualdad demográfica y económica entre los municipios; los problemas de salud cruzan las fronteras municipales; y la necesidad de tener tantos apa- ratos administrativos como comunas hay crea un derroche continuo de recursos, una gran ineficiencia económica ${ }^{7}$. Sólo puede comprenderse la insistencia del Ministerio de la época en promulgar el Estatuto, por la influencia en algunos directivos del ejemplo de realidades extranjeras como la de ciertas comunas de EEUU o de Europa, donde hay tradición y capacidad de autogobierno local, por un lado, y ausencia de un sistema público nacional de salud como el chileno, por otro. También podría explicarse por la necesidad de exhibir una reducción del tamaño del Estado central: el "ajuste estructural" recomendado por el Banco Mundial $^{7}$. El argumento de que la administración municipal de los consultorios favorece la descentralización efectiva y la participación de la población no era sostenible a la luz de la situación de entonces ni lo es ahora: después de 20 años del regreso a la democracia, las direcciones de salud de la mayoría de las municipalidades de Santiago informan que cuentan con sólo un estrecho margen de flexibilidad en el cumplimiento de los requisitos programáticos diseñados por el ministerio; y muchas carecen del apoyo de comunidades que participen activamente en los Consejos de Desarrollo de los consultorios o en otras instancias.

Tabla 4. Mortalidad infantil de Chile, 2002-2008.

\begin{tabular}{|c|c|c|c|}
\hline Año & $\begin{array}{c}\text { M. Infan- } \\
\text { til }\end{array}$ & M. Neonatal & $\begin{array}{c}\text { M. post- neo- } \\
\text { natal }\end{array}$ \\
\hline 2002 & 8,1 & 5,2 & 2,9 \\
\hline 2003 & 8,2 & 5,1 & 3,1 \\
\hline 2004 & 8,7 & 5,6 & 3,1 \\
\hline 2005 & 8,2 & 5,4 & 2,8 \\
\hline 2006 & 7,9 & 5,4 & 2,5 \\
\hline 2007 & 8,3 & 5,6 & 2,7 \\
\hline 2008 & 7,8 & 5,5 & 2,3 \\
\hline
\end{tabular}

Fuente: INE, Demografía 2010. 
Tabla 5. Algunos indicadores nacionales y regionales del sistema público de atención de salud.- Chile 2009*

\begin{tabular}{|c|c|c|c|c|c|c|c|c|}
\hline Indicador * & País & Mejor región & $\begin{array}{c}\text { Peor } \\
\text { región }\end{array}$ & $\begin{array}{c}\text { R. Metro- } \\
\text { politana }\end{array}$ & $\begin{array}{c}\text { R. Val- } \\
\text { paraíso }\end{array}$ & R. Biobío & $\begin{array}{c}\text { R. Arau- } \\
\text { canía }\end{array}$ & R. Ríos \\
\hline $\begin{array}{c}\text { \% Cobertura } \\
\text { efectiva HTA }\end{array}$ & 7,3 & 10,3 & 3,4 & 6,4 & 8,2 & 8,8 & 9,1 & 7,2 \\
\hline $\begin{array}{c}\text { \% Cobertura } \\
\text { efectiva Dia- } \\
\text { betes }\end{array}$ & 2 & 3,1 & 1,1 & 1,6 & 2,6 & 3,1 & 2,3 & 2,2 \\
\hline $\begin{array}{c}\text { \% Prevalencia } \\
\text { discapacidad }\end{array}$ & 12,9 & 6,9 & 17,8 & 11,6 & 8,5 & 15,1 & 17,6 & 14,4 \\
\hline $\begin{array}{c}\text { \% Pap } \\
\text { vigente }\end{array}$ & 63,1 & 67,1 & 54,7 & 63,9 & 64,1 & 63,1 & 62 & 67,1 \\
\hline $\begin{array}{c}\text { \% menores } \\
\text { de 6 años en } \\
\text { control }\end{array}$ & 69,1 & 90,3 & 47,3 & 60,6 & 78,4 & 77,4 & 81,4 & 77,8 \\
\hline
\end{tabular}

* Departamento de Epidemiología, Ministerio de Salud. Se especifican las regiones en que hay becados del Programa.

\section{EL IMPACTO EN EL STATUS DE LAAPS Y EN LOS MÉDICOS}

El Estatuto de la APS municipalizada significó la garantía de mejores remuneraciones para algunos estamentos. Produjo, sin embargo, un alejamiento de los médicos, para los cuales es importante contar con una carrera profesional que integre la perspectiva de especialización y de trabajo en hospital. Ello derivó en la escasez de médicos en la APS; en la frecuente rotación de ellos; en una selección negativa en cuanto a capacidad técnica $\mathrm{y}$, como compensación, en tener una alta proporción de médicos formados fuera del país. Aún hoy, en las comunas de Santiago, hay municipalidades que necesitan emplear a personal que no ha validado su título de médico. Otra consecuencia fue la desvalorización del trabajo médico en consultorios, en comparación con su status en los hospitales u otros sitios de trabajo. Tal situación se ha asociado a la polarización de la función médica hacia la satisfacción de la demanda espontánea por atenciones curativas en desmedro de la promoción y prevención y de la continuidad de la relación médico-paciente. La desvalorización de la labor médica en la APS se ha extendido a la atención primaria en general y hasta cierto punto, al sector público de atención de salud. La población, aunque valora altamente la atención que logra recibir de los médicos, está insatisfecha con el sector en general. Los gobiernos han reaccionado aumentando el control administrativo y económico de la "producción" de prestaciones, definidas en una forma fragmentada, ajena a la naturaleza de la atención a las personas.

En los años 90 se propuso paliar la falta de médicos de la APS con la creación de cargos de "médicos integrales" y con la formación de "médicos de familia"8. Estas medidas no han dado los efectos deseados. La situación descrita ha sido agravada por la atracción del ejercicio de la profesión médica en el sector privado, y por la falta o poca utilización de especialistas en salud pública, que pudieran hacer, como les corresponde por su formación, una verdadera síntesis entre la clínica, la administración y la epidemiología. La legislación dictada en el año 2004 consagró la división entre salud pública y asistencia; entre los hospitales -por una parte- y los municipios, con los consultorios, por otra parte; entre servicios de salud y secretarías regionales ministeriales. La atención primaria de salud dejó de tener un pensamiento propio, integrado en una doctrina sanitaria 
nacional y que contribuyera a una política nacional de salud. La Declaración de Alma Ata, la Carta de Ottawa y sus sucesoras dejaron de tener vigencia real en Chile, a pesar de que expresaban lo que había sido la práctica y la enseñanza del Servicio Nacional de Salud y sus antecesores.

\section{PARA UNA APS RESOLUTIVA Y UN SISTEMA INTEGRADO: EL PROGRAMA DE FORMACIÓN EN LAS ESPECIALIDADES BÁSICAS}

En estas circunstancias, se planteaba con claridad y urgencia crecientes la necesidad de una estrategia que permitiera fortalecer el pilar central del sistema público de atención: la atención primaria de salud. Se requería darle mayor resolutividad e integración en los aspectos curativo, promocional y preventivo; hacer que los pacientes transitaran de manera fluida hacia y desde los hospitales cuando esto fuera necesario; y lograr cobertura y efectividad adecuadas; y como resultado de lo anterior, aumentar su prestigio en la comunidad nacio- nal. La estrategia consistiría en atraer a la APS a los médicos egresados de las facultades de medicina de universidades chilenas acreditadas. Para ello, se usaría lo esencial de la experiencia del SNS, antes descrita: en un plan de seis años, trabajarían en los consultorios, alternando con períodos de formación en las especialidades básicas, que les darían capacidad resolutiva y los conducirían al título de especialista. A ello se agregaría una elaboración continuada de la experiencia en el marco de los conceptos y técnicas de la Salud Pública. Se esperaba que algunos de dichos médicos siguieran actuando en la APS, especialmente si se elaboraba una política apropiada de recursos humanos de parte del Ministerio9.

Después de diversos intentos, el Programa fue convenido en 2006-2007 por el Ministerio y la Universidad de Chile, con participación de varias municipalidades, y con mucho interés de los estudiantes. La expansión que ha tenido lugar en los años de ejecución -2008 al actual, 201- se presenta en las Tablas 1 a la 3.

En el año 2011 los becados de la Universidad de Chile están activos en cuatro campus, con siete hospitales, y en 10 comunas, con 20

Tabla 6. Cobertura de la población beneficiaria del sistema público de atención de salud con examen médico preventivo (EMPA). Chile, Región Metropolitana. 2006-2008.

\begin{tabular}{|l|l|c|c|c|}
\hline \multirow{2}{*}{ Servicios de Salud } & \multirow{2}{*}{ Sexo } & \multicolumn{3}{|c|}{ Tasa anual } \\
\cline { 3 - 5 } & & $\mathbf{2 0 0 6}$ & $\mathbf{2 0 0 7}$ & $\mathbf{2 0 0 8}$ \\
\hline Norte & & 11,99 & 12,41 & 12,41 \\
\hline Occidente & & 13,11 & 12,37 & 14,93 \\
\hline Central & & 5,42 & 4,45 & 8,47 \\
\hline Oriente & & 11,74 & 15,93 & 16,1 \\
\hline Sur & & 11,68 & 12,14 & 14,42 \\
\hline Sur-Oriente & & 13,94 & 9,69 & 10,75 \\
\hline & & & & \\
\hline REGIÓN & Hombres & 4,37 & 5,01 & 6,87 \\
\hline & Mujeres & 18,81 & 18,56 & 18,57 \\
\hline & Ambos sexos & 11,63 & 11,82 & 12,65 \\
\hline
\end{tabular}

Fuente: Peña V, Examen Médico Preventivo (EMP). Coberturas y factores asociados a su aplicación, Cuad Med Soc $2009 ; 49: 163-70$ 
consultorios. Con los becados de otras siete universidades se completa la presencia en 21 comunas.

\section{PRIMEROS APRENDIZAJES}

La experiencia ha ido mostrando diversos aspectos de la realidad de la APS, particularmente en relación con el papel de los médicos en ella. Más allá de la estructura del Programa y de sus guías iniciales, se ha logrado una comprensión más profunda y detallada de los problemas que hay que resolver para centrar la atención en las personas, las familias y la comunidad. Junto con los médicos becados, se penetró bajo el diagnóstico inicial y los objetivos consiguientes a lograr -que se resumieron más arriba- y se definieron doce problemas y doce objetivos nuevos o "de segundo orden"10. Brevemente enunciados, estos objetivos son:

- Integrar a los médicos a la organización, equipos y actividades de cada centro de salud.

- Lograr una adecuada relación médico-paciente.

- Obtener una participación activa de los médicos becados en las acciones de promoción y prevención.

- Hacer realidad la acción de los becados en la comunidad y con autoridades y agencias sectoriales del área asignada al centro.

- Implementar efectivamente la dimensión familiar de la atención.

- Adecuar la normatividad, la organización y la conducción de los centros de modo de facilitar el funcionamiento de los equipos en torno a los pacientes, las familias y los grupos.

- Elevar el nivel de conocimientos, destrezas y actitudes de los médicos generales y de otros profesionales y técnicos de los centros, en lo que se revelara necesario.

- Contribuir a coordinar el funcionamiento del subsistema o red local de atención.

- Adecuar el uso de los medicamentos y de los exámenes indicados.

- Fortalecer la supervisión de la función clí- nica en la APS por los médicos de los Departamentos universitarios y de los servicios hospitalarios.

- Mejorar la efectividad de los centros participantes mediante la comparación de sus actividades y de los factores que influyen en ella

- Evaluar el efecto del sistema sobre el estado de salud de la población asignada.

Se identificaron indicadores para evaluar la consecución de estos objetivos. Esta evaluación está en curso ininterrumpido y es hecha por los propios médicos en formación, con el apoyo de docentes y tutores.

\section{HACIA CONSULTORIOS FORTALECIDOS EN LAS FUNCIONES DE CURACIÓN, PROMOCIÓN Y PREVENCIÓN}

Ya antes se vio la necesidad de hacer posible la diversificación del papel de los "médicos especialistas en formación" (EEF) mediante la destinación de una parte del tiempo contratado a funciones que no fueran directamente asistenciales sino que se orientaran a algunos de los “objetivos de segundo orden". Ello se especificó en un breve documento que se anexó al Convenio del Programa entre los Servicios de Salud y los Municipios (“el anexo 1"), y que ha sido útil para evitar una orientación exclusivamente curativa y dedicada al rendimiento cuantitativo. El mismo anexo ha servido para ir hacia la focalización gradual del trabajo asistencial de los médicos becados hacia los pacientes de su especialidad.

La experiencia de estos años nos ha demostrado a todos los participantes cómo la tarea del Programa debe ser fortalecer a la APS en general y en cada centro de salud en que hay especialistas en formación. Por ejemplo, ellos pueden actuar, y actúan, como consultores para los otros profesionales al interior del consultorio; pueden hacer capacitación de ellos formalmente, o bien informalmente, a través de reuniones de médicos o de "sector". Mejo- 
ran grandemente la calidad de las derivaciones al especialista y pueden resolver de manera inmediata las trabas que impiden o retardan una interconsulta, la realización de un examen o la disponibilidad de un medicamento. El apoyo de los tutores clínicos incorporados progresivamente al Programa refuerza la capacidad, la seguridad y la autoridad de los EEF. Al racionalizar los procesos de diagnóstico y tratamiento, se generan economías para el centro. Cada vez se percibe más claramente cómo podrán producir eficiencia al disminuir el trabajo de los servicios de urgencia y de los consultorios de especialidades y de las propias salas de hospital, a través de tratamientos correctos y oportunos.

\section{CLARIFICACIÓN DE FUNCIONES PARA UNA MEJOR INTEGRACIÓN EN LOS EQUIPOs}

Se hizo necesario explicitar estos propósitos y estos efectos mediante una exposición aún más detallada de las funciones de los EEF. Este documento fue distribuido en reuniones de cada comuna en marzo de 2011 y ha sido publicado ${ }^{11}$. En su carácter de "nuevo anexo 1 ", constituye un instrumento que ayuda a clarificar las funciones del médico entre las demás de los miembros del equipo; y así, en vez de aislar a dicho profesional en una labor monotónica de despachador de la "morbilidad", lo integra a la estructura y las funciones del consultorio. Eventualmente, lo integrará a la comunidad que sirve.

\section{LASITUACIÓN REAL DE LAATENCIÓN PRIMARIA DE SALUD Y DE LA SALUD}

La debilidad de la APS -pilar del sistema de atención- en años recientes estaba de manifiesto. El descenso de la mortalidad infantil aparecía detenido en una tasa que fluctuaba entre 7 y 8 por mil NV, mientras en otros países del continente progresaba más nítidamente
(Tabla 4).

La encuesta de salud de 2009-10 mostró que el país no está controlando aquellos factores negativos cuya reducción depende de políticas nacionales que en gran parte deben ser ejecutadas por la APS. La prevalencia del tabaquismo permanece sobre el $40 \%$; la del beber problema, sobre el 55\%; el sedentarismo afecta al $89 \%$ de la población; lo síntomas depresivos afectan al $17 \%$; el consumo de sodio pasa desapercibido, a un nivel de 9,8 gramos diarios de sal. El exceso de peso subió de $61 \%$ a $67 \%$ entre 2003 y 2010 y la obesidad mórbida se duplicó 11. La hipertensión arterial y la diabetes tienen una prevalencia alta en la población más desfavorecida, por la cual debería responder la APS, y la cobertura no se asocia a una tasa de compensación suficiente, como se aprecia en el indicador "cobertura efectiva" que se informa en el país y en sus regiones (Tabla 5$)^{13}$.

Frente al predominio de las enfermedades crónicas del adulto, la estrategia más poderosa a usar por la APS es el Examen de Medicina Preventiva del Adulto de 20 a 64 años. Sin embargo, la cobertura alcanzada en la Región Metropolitana era apenas del $12,65 \%$ en el 2009 , y en los varones, que serían los más beneficiados, no alcanzaba al $7 \%$ (Tabla 6$)^{14}$. Además, falta especificar claramente la periodicidad adecuada para el examen y normar los métodos de captación, seguimiento y re-captación.

La situación descrita es consistente con lo observado en los centros de salud de la capital. Sin perjuicio de que se completará un perfil de aquellos con los cuales se trabaja en el Programa, impresiona desde ya la diferencia observado entre ellos, a la luz de algunos indicadores. Entre 17 centros, la población validada por el Fonasa fluctuaba, en el 2010, entre 17.730 y 48.121 beneficiarios; el personal total, entre 1,5 y 3,1 por mil beneficiarios; las horas semanales contratadas de médicos no becados, entre 43 y 179 por 10.000 beneficiarios. En algunos casos falta un programa estructurado de adolescencia o de la mujer; en otros, no se mide la satisfacción de los usuarios. Es poco 
frecuente que se realicen estudios multidisciplinarios de familias o de barrios. No en todos se pueden realizar procedimientos diagnósticos o terapéuticos instrumentales simples. En la mayoría, el espacio es insuficiente. A falta de facultades para pensar y planificar localmente, los centros se ven restringidos a usar métodos, prioridades y metas definidos centralmente y vinculados a estímulos o sanciones económicas institucionales e individuales.

\section{PREOCUPACIONES. HACIA UNA ATENCIÓN VERDADERAMENTE PREVENTIVA Y VERDADERAMENTE CURATIVA. EL ENFOQUE DE SECTOR: POBLACIONAL Y TERRITORIAL}

Los EEF se inquietan por la impotencia para hacer prevención primaria o secundaria de las enfermedades crónicas no trasmisibles, o para postergar su aparición o sus secuelas. La respuesta para ello está en que los equipos multidisciplinarios, médicos incluidos, conozcan, mapa en mano, cada cuadra de sus sectores asignados, y a todas las familias que ahí viven. Esta es la estrategia que en el tiempo del SNS permitió controlar la mortalidad y morbilidad materno-infantil: saber qué niño estaba desnutrido y no había venido a control, saber a quiénes les faltaba alguna dosis de vacuna, a quiénes debía persuadirse para ingresar a un Grupo de Alcohólicos Recuperados. Hoy día se logrará efectividad y eficiencia si se conocen los problemas y condiciones por los cuales cada familia completa tendría que ser atendida de manera integrada por el consultorio; y si se puede hacer el EMPA de cada persona con la periodicidad requerida. El Programa de los EEF está en el umbral de abordar esta estrategia. Frente al que ya es un enfermo crónico, se entiende la síntesis entre la vida y la enfermedad, y que no morirán de hipertensión o de diabetes, sino de vivir con hipertensión o con diabetes, controlada o no (es un modo de vivir), y que lo importante, el objetivo del autocuidado y del cuidado es mantener las funciones de moverse, de ver, de oír, las fa- cultades de pensar, de sentir, de estar seguro; o de saber compensar las funciones disminuidas con otras.

Aparecen otros interrogantes de los EEF. ¿Cómo captar para la prevención al hombre adulto que se siente sano y que trabaja lejos del barrio? El sistema, la APS, debe adaptarse. El centro de salud debe atender a quien trabaja en el sector, aunque resida en otra comuna; debe hallarse una solución al tema de la inscripción Fonasa y a la adjudicación de la asignación "per capita" del trabajador y de su familia, o al mejor aprovechamiento de la cotización patronal por accidentes y enfermedades laborales.

La presencia de los EEF permite la realización de policlínicos en el consultorio: no sólo de IRA, también de SCV, o de psiquiatría, de reumatismo u obesidad ${ }^{15}$. Son una ventaja para el paciente y disminuyen las esperas y las interconsultas. Se organizan ahí donde hay colaboración del especialista y de la dirección del consultorio, y / o mucho empeño del becado. Más adelante podrá realizarse la idea de reemplazar los "policlínicos-problema" por "policlínicos-persona" o "policlínicos-grupo". A propósito del apoyo del director, hemos aprendido que importa más su personalidad que su profesión, y que el impacto que tiene sobre la efectividad de todos es muy significativo.

\section{EL PROGRAMA ES IMPORTANTE. Y HASTA EL PRÓXIMO COMUNICADO}

En la fase actual del desarrollo del Programa, valoramos el potencial del mismo como factor visible de Equidad, con compromiso del Estado. Y apreciamos el apoyo transversal recibido. Una prueba decisiva se tendrá en los años 2014 a 2017, cuando se titulen lo EEF que hoy están en el Programa y el Ministerio tenga la oportunidad de integrarlos en una política y plan de personal que sea la continuación coherente de los principios que han inspirado su formación y que proclamaron cuando eran estudiantes.

No cabe duda de que podremos seguir co- 
municando lo que se vaya cosechando en el aprendizaje continuo que tiene lugar en esta escuela de atención primaria que es el Programa de los EEF.

Reconocimiento: a los diversos equipos que están haciendo realidad el Programa en sus varias facetas, y a los jóvenes médicos participantes.

Conflicto de interés: ninguno.

\section{REFERENCIAS}

1. MOLINA G. Orientaciones de la Salubridad, Revista Chilena de Higiene y Medicina Preventiva 1945; 7(4): 251-307. Reproducido en: Cuad Med Soc 2005; 45: 62-68

2. HORWITZ A. Medicina en Chile, Revista del Servicio Nacional de Salud 1956; 1 (1): 3-4.

3. VALENZUELA G, HORWITZ A, Doctrina del Servicio Nacional de Salud, en : Revista del SNS 1956; 1 (1):5-18

4. DURÁN H, SOLAR M. La organización local del SNS, en Revista del SNS 1956 1(1): 19-22. Extractos reproducidos en Cuad Med Soc 2005; 45: 163170

5. MONTOYA-AGUILAR C, Atención médica del niño, en: Meneghello j et al (eds.), Pediatría, Editorial Inter.-Médica, Bs. Aires 1972. Capítulo reimpreso, Departamento de Extensión de la Universidad de Chile, Santiago, 1972.

6. CARVAJAL Y. La experiencia de medicina preventiva integrada a la clínica. Hospital San Francisco de Borja 1963-1973, Cuad Med Soc 2009;29 (2): 101-108
7. Numerosos autores institucionales e individuales, Jornada sobre la Salud Municipalizada. Cuad Med Soc 1992; 33 (2): 3-70

8. MARGOZZINI J ET AL. Evaluación del programa de médicos generales urbanos en el Area Metropolitana Norte, Cuad Med Soc 1978; 19 (4): 22-30

9. IPINZA M. Como solucionar el déficit de médicos en los Consultorios urbanos municipalizados, Cuad Med Soc 2004; 44 (2): 73-80

10. MONTOYA-AGUILAR C. La atención primaria de salud en Chile y el papel de los médicos. Nuevos objetivos en el año 2010, Cuad Med Soc 2010; 50 (3): 167-177

11. MONTOYA-AGUILAR C, VARGAS N. Organización de la atención primaria de salud urbana, con incorporación de especialistas en formación. Cuad Med Soc 2011; 51 (1): 40-47

12. Subdepartamento de Epidemiología. Departamento de Salud Pública. Seremi de Salud Metropolitana (2011). Encuesta Nacional de Salud de Chile, 20092010. Indicadores de la Región Metropolitana. Santiago.

13. Departamento de Epidemiología. Ministerio de Salud de Chile (2009). Diagnósticos regionales de salud con enfoque de determinantes sociales. Santiago.

14. PEÑA V. Examen médico preventivo (EMP). Coberturas y descripción de los factores asociados a su aplicación en población usuaria del Sistema Público de Salud en la Región Metropolitana. Cuad Med Soc 2009; 49 (3): 163-70.

15. ARAVENAP, RUIZ P. Experiencia en la implementación de un policlínico de choque de salud mental en un establecimiento de atención primaria. Cuad Med Soc 2011; 51 (1): 5-15

Usted puede comentar éste y otros artículos publicados en la Revista Chilena de Salud Pública, enviando un correo electrónico a revistasp@med.uchile.cl 